\title{
ESTUDIO COMPARATIVO SOBRE LA CULTURA AMBIENTAL EN NIÑOS Y NIÑAS DE 5 AÑOS DE HUANCAYO, PERÚ
}

\author{
Abdías Chávez-Epiquén* \\ https://orcid.org/0000-0001-5589-5217 \\ Julia Teves-Quispe** \\ https://orcid.org/0000-0002-7009-9937 \\ María Jesús López Verde*** \\ https://orcid.org/0000-0003-4886-1136
}

RECIBIDO: Diciembre 2019 / ACEPTADO: Mayo 2020 / PUBLICADO: Septiembre 2020

Como citar: Chávez-Epiquén, Abdías; Teves-Quispe, Julia; López Verde, María Jesús. (2020). Estudio comparativo sobre la cultura ambiental en niños y niñas de 5 años de Huancayo, Perú. Telos: revista de Estudios Interdisciplinarios en Ciencias Sociales, 22 (3), Venezuela. (Pp.528-540).

DOI: www.doi.org/10.36390/telos223.04

\section{RESUMEN}

El presente artículo tuvo como objetivo comprobar si existen diferencias significativas en la percepción y conocimiento de los menores de 5 años provenientes de los Distritos de Ingenio y Saño - Huancayo, Perú, 2017 hacia la cultura ambiental, considerando las teorías de ÁngelMaya (2003), Hernández-Ayón y Hernández-Ayón (2014), Fishbein y Ajzen (1975), Ajzen y Fishbein (1980), Ajzen y Madden (1986), Álvarez y Vega (2009). La investigación, por su naturaleza fue de tipo básico teórica y de modo específico se utilizó el método cuantitativo, con un diseño descriptivo comparativo. Se empleó la técnica evaluación pedagógica y como instrumento la lista de cotejo, con 20 ítems sobre las variables de cultura ambiental. Se trabajó con una muestra de 15 niños y 15 niñas de educación preescolar de cada distrito. Se aplicó una evaluación pedagógica y una lista de cotejo, sobre las cualidades hacia cultura ambiental. Se evidenció la presencia de diferencias significativas de percepción hacia la formación ambiental en niños y niñas de 5 años, con un nivel de significancia de $0,05 \mathrm{y} t=2,198$; con lo que demuestra una ligera desventaja en los niños del distrito de Ingenio con respecto al distrito de Saño. Se considera necesario destinar todos los medios posibles y voluntades para que los profesionales en educación ambiental puedan dirigirse a los niños de manera más efectiva.

Palabras clave: Niños, actitud, cultura ambiental, educación ambiental, conciencia ambiental.

* Doctor en Educación. Universidad Nacional Ciro Alegría. Perú. Correo electrónico: achavez@unca.edu.pe

** Doctora en Educación. Universidad Nacional Mayor de San Marcos. Perú. Correo electrónico: jtevesq@unmsm.edu.pe

*** Magister en Gestión pública, Lcda. Trabajo Social. Universidad César Vallejo. Perú. Correo electrónico: marialopezverde@hotmail.com 


\title{
Comparative study on environmental culture in 5-year-old children in Huancayo, Peru
}

\begin{abstract}
The present article aimed at verifying if there are significant differences in the perception and knowledge of children under 5 years old coming from the Districts of Ingenio and Saño Huancayo, Peru, 2017 towards environmental culture, considering the theories of Angel-Maya (2003), Hernández-Ayón and Hernández-Ayón (2014), Fishbein and Ajzen (1975), Ajzen and Fishbein (1980), Ajzen and Madden (1986), Álvarez and Vega (2009). The research, by its nature was of a basic theoretical type and specifically used the quantitative method, with a descriptive comparative design. The pedagogical evaluation technique was used and as an instrument the checklist, with 20 items on the variables of environmental culture. We worked with a sample of 15 boys and 15 girls in preschool education in each district. A pedagogical evaluation and a checklist were applied, on the qualities towards environmental culture. The presence of significant differences of perception towards environmental education was evidenced in boys and girls of 5 years old, with a level of significance of 0.05 and $t=2.198$; which shows a slight disadvantage in the children of the district of Ingenio with respect to the district of Saño. It is considered necessary to allocate all possible means and wills so that environmental education professionals can address children more effectively.
\end{abstract}

Keywords: Children, attitude, environmental culture, environmental education, environmental conscience.

\section{Introducción}

La cultura ambiental según De Alba et al (1993), representa como está conformada la vida de los individuos, simbolizada además por las diferentes formas de nexos que estos han mantenido con su ambiente natural según la historia, según sus necesidades básicas para subsistir como alimentación y salud entre otros, así como admirar su belleza paisajística y conocer además los beneficios que este le puede concebir.

Por su parte para Bayon y Morejon (2005), este término está sostenido por el vínculo que existe entre los individuos y su medio ambiente; relación que involucra a una variedad de estilos, tradiciones y circunstancias de vida de una colectividad, con características propias basada en su historia cultural.

Esta se define además como acervo y elemento de los ecosistemas; en donde, su protección es un derecho por excelencia de las comunidades, por lo que cuando el sistema está compuesto de buenos elementos que le permiten cubrir sus necesidades básicas y generar el uso razonable de todos los recursos naturales, fundamentados en las necesidades de cada comunidad, observándose además que la sociedad cambia su rumbo hacia el desarrollo sostenible de su ambiente (Roque 2003).

Del mismo modo, Ángel-Maya (1995), bajo la visión antropológica de la palabra, este la define como la manera distintiva que presenta cada grupo de individuos para adaptarse a su entorno. Esta base de acoplamiento (la cultura), además de considerar los diferentes instrumentos para desarrollarla, considera además las diferentes maneras como se estructura 
la sociedad y como esta se relaciona con los sistemas sociales. Para Ángel-Maya (2003), es necesario considerar para el desarrollo de una buena formación ambiental las diferentes maneras en las cuales la sociedad se organiza y articula a través de estrategias adaptativas.

Algunos investigadores como Hernández-Ayón y Hernández-Ayón (2014), perciben a la cultura como un estilo de vida, estructurado por la manera en como se ha visto afectada la sociedad por el modelo económico de cada país, creando una cultura ambiental con intereses, opiniones, comportamientos y conductas basados en una sociedad o cultura insustentable, y por demás difícil de satisfacer generando así muchas veces daños ecológicos.

En el estudio de las actitudes ambientales humanas fueron los investigadores Fishbein y Ajzen (1975), quienes desarrollaron un método para relacionar las actitudes o cualidades, creencias, y el comportamiento, conocidos como modelo de valor-expectativa, lo que posteriormente dio pie a la creación de la teoría de la acción razonada Ajzen y Fishbein (1980) y, posteriormente a la conocida teoría de la acción planificada (Ajzen y Madden, 1986).

Estos patrones sobre cualidades humanas han sido de gran influencia para las diferentes cualidades y comportamientos sostenibles con gran repercusión en una formación ambientalista bajo la visión de la Psicología Ambiental, para así instaurar las relaciones entre el comportamiento habitual hacia el ambiente y las conductas que se ponen en manifiesto a través del tiempo Álvarez y Vega (2009). Bajo esta misma visión, Holahan (2014), las definió como la disposición emocional optimista o perjudicial que se puede presentar alguna peculiaridad del medio o hacia alguna situación problemática relacionado con él.

Con respecto a la educación ambiental en las ciencias sociales se han generado acercamientos epistemológicos para desarrollar sociedades basadas en un comportamiento sustentable, reconociéndose la importancia del comportamiento personal y de la manera que se actúa con la comunidad en momentos donde se afecta la salud del medio ambiente (Maldonado et al 2011; Severiche-Sierra y Acevedo, 2013, Bleys, et al 2018).

A pesar de todas las estrategias puestas en práctica a través del tiempo para mantener en buen estado las riquezas que provienen de la naturaleza ha sido infructuoso disminuir o crear grandes beneficios en contra de la destrucción ambiental, debido, probablemente a que se ha logrado obtener una sociedad con una conciencia de alto nivel enmarcado en el respeto hacia los ecosistemas que les rodea (Severiche-Sierra et al., 2016).

El presente estudio se orienta hacia la línea de responsabilidad social, con el fin de determinar las diferencias significativas de cualidades entorno a la formación ambiental de individuos de 5 años de los distritos de Ingenio y Saño, Huancayo, Perú". Considerando que la preservación del ambiente es un tema mundial que atañe al desarrollo de la humanidad en contacto con la naturaleza, de tal forma que existen principios y leyes que reglamentan para el adecuado uso de recursos naturales. De todas estas funciones, el protagonista es el hombre como único responsable de administrar, preservar, educar y formar una cultura hacia el cuidado de nuestros recursos.

El objetivo de la investigación fue comprobar si existen diferencias significativas en la percepción y conocimiento de los menores provenientes de los Distritos de Ingenio y Saño Huancayo, Perú, 2017 hacia la cultura ambiental enmarcados en actitudes más humanas, con valores ambientales, control de ruidos, comunicación ambiental. 


\section{Metodología}

Se realizó un estudio básico teórico bajo el método cuantitativo, con un esquema descriptivo comparativo. Se empleó la técnica de evaluación pedagógica y la lista de cotejo como instrumento a aplicar, estructurado en 20 ítems sobre las variables de cultura ambiental.

Se aplicó un diseño descriptivo comparativo con dos muestras para así evaluar las cualidades y determinar las diferencias significativas entre los individuos en estudio de 5 años, procedentes de los distritos de Ingenio y Saño de la provincia de Huancayo, Perú.

El diseño propuesto se basó en el diseño descriptivo comparativo, según Sánchez y Reyes (2015) quienes consideran que este diseño "está orientado a la descripción y comparación de dos o más muestras independientes". (p. 79). El esquema es:

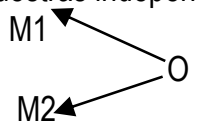

Donde:

O: Observación de la variable actitud hacia la cultura ambiental

M1: Muestra niños y niñas de 5 años de los distritos de Saño

M2: Muestra niños y niñas de 5 años de los distritos de Ingenio

Se trabajó con una muestra de 15 niños y 15 niñas de 5 años que presentaran actitudes cognoscitivas típicas de niños de su edad a través habilidades y destrezas desarrolladas por medio de experiencias y aprendizajes para adaptarse al medio; los cuales eran provenientes de las instituciones públicas Institución Educativa modalidad inicial jardín (I.E.) N 355 de Saño con 54 niños, fundada en el año 2008 y la Institución Educativa modalidad inicial jardín (I.E.) N ${ }^{\circ} 400$ de Ingenio con 40 niños, fundada en el año 2010, estas fueron escogidas por ser unas de las que contaban con más tiempo de experiencia en el área educativa de la zona y por contar con mayor número de niños entre las edades de interés para el estudio.

Se diseñó una prueba de cualidades posteriormente validada por expertos en el área. La fiabilidad del instrumento se valoró por medio de la aplicación de un experimento piloto y posteriormente comparada por medio del Alfa Cronbach.

El instrumento fue aplicado con el fin de reconocer si hay o no desigualdades en las cualidades hacia la cultura ambiental, entre ellas las humanas, los valores ambientales y el control de ruidos en los niños y niñas de 5 años del distrito de Ingenio y Saño - Huancayo, Perú. 2017.

\section{Resultados y Discusión}

Para la prueba de confiabilidad del instrumento aplicado se obtuvo un Alfa de Cronbach de 0.897 demostrando un alto grado de confiabilidad.

Según las características de las variables y dimensiones estudiadas, se obtuvo una distribución homogénea en los niños de la I.E. N ${ }^{\circ} 400$ de Ingenio y muy dispersa en los niños de la I.E. N ${ }^{\circ} 355$ de Saño.

Se observó que de los 30 alumnos evaluados el $40 \%$ de la muestra presentó un nivel excelente (12) provenientes del distrito de Saño y un $16.66 \%$ (5) provenientes del distrito Ingenio, observándose una elevada diferencia en la formación ambiental entre los niños provenientes de los dos distritos en estudio (tabla 1). 
Tabla 1. Prueba de actitud hacia la cultura ambiental aplicada a niños y niñas de 5 años provenientes de los Distritos Ingenio y Saño, Huancayo, Perú.

Fuente: elaboración propia

\begin{tabular}{ccc}
\hline Nivel & Número de estudiantes (\%) & Distritos \\
\hline Excelente & $12(40 \%)$ & Saño \\
& $5(16.66 \%)$ & Ingenio \\
\hline Bueno & $2(6.66 \%)$ & Saño \\
& $5(16.66 \%)$ & Ingenio \\
\hline Regular & $0(\%)$ & Saño \\
& $5(16.66 \%)$ & Ingenio \\
\hline Deficiente & $1(3.33 \%)$ & Saño \\
& $0(0 \%)$ & Ingenio \\
\hline
\end{tabular}

Según la medición de tendencia central la media aritmética de las cualidades hacia la formación ambiental en los individuos de la I.E. 355 de Saño fue 18, mientras que los de la I.E. $\mathrm{N}^{\circ} 400$ del distrito de Ingenio la media fue de 16. La desviación estándar en los niños de la I.E. de Saño fue 2.76, y en los niños de Ingenio la desviación fue 2.18. La mediana en los niños de Saño fue 19 y para los niños de Ingenio fue 16. La moda en los niños de Saño fue 19 y en los niños de Ingenio fue 18.

El puntaje máximo en los niños de Saño fue 20 y el mínimo 9, mientras que en los niños de Ingenio el máximo fue 19 y el mínimo 13.

Siguiendo los procedimientos, métodos y técnicas de rigor científico y del trabajo de campo, se obtuvo como resultado la diferencia de medias de los grupos de trabajo: Grupo 1 I.E. $N^{\circ} 355$ de Saño y del Grupo 2 I.E. N ${ }^{\circ} 400$, se confirma que existe diferencias significativas entre las cualidades hacia la formación ambiental en los individuos de 5 años de los distritos en estudio (tabla 2).

Tabla 2. Resultados del T student sobre las cualidades hacia la formación ambiental en niños y niñas de 5 años de los Distritos Ingenio y Saño, Huancayo, Perú.

\begin{tabular}{|c|c|c|}
\hline Valores & Distrito de Saño & Distrito de Ingenio \\
\hline Media aritmética & 18.06 & 16.06 \\
\hline T Student & 2.198 & 2.198 \\
\hline P & 0.00 & 0.00 \\
\hline Nivel de significancia & 0.05 & 0.05 \\
\hline
\end{tabular}

Fuente: elaboración propia

Se comprobó que existen diferencias significativas en las cualidades humanas en niños y niñas de 5 años de los distritos Ingenio y Saño - Huancayo 2017 (tabla 3). 
Tabla 3. Resultados del T student sobre las cualidades humanas en niños de 5 años de los Distritos Ingenio y Saño, Huancayo, Perú.

\begin{tabular}{|c|c|c|}
\hline Valores & Distrito de Saño & Distrito de Ingenio \\
\hline Media aritmética & 16.33 & 15.05 \\
\hline T Student & 3.136 & 3.136 \\
\hline$p$ & 0.004 & 0.004 \\
\hline Nivel de significancia & 0.05 & 0.05 \\
\hline
\end{tabular}

Fuente: elaboración propia

Se comprobó que existen diferencias significativas de los valores ambientales en niños y niñas de 5 años de los distritos Ingenio y Saño - Huancayo 2017 (tabla 4).

Tabla 4. Resultados del T student sobre valores ambientales en niños de 5 años de los Distritos Ingenio y Saño, Huancayo, Perú.

\begin{tabular}{|c|c|c|}
\hline Valores & Distrito de Saño & Distrito de Ingenio \\
\hline Media aritmética & 14.2 & 12.2 \\
\hline T Student & 2.389 & 2.389 \\
\hline$p$ & 0.024 & 0.024 \\
\hline Nivel de significancia & 0.05 & 0.05 \\
\hline
\end{tabular}

Fuente: elaboración propia

Se observó diferencias significativas en el control de ruidos en niños y niñas de 5 años de los distritos Ingenio y Saño - Huancayo 2017 (tabla 5).

Tabla 5. Resultados del T student sobre el control de ruidos en niños de 5 años de los Distritos Ingenio y Saño, Huancayo, Perú.

\begin{tabular}{|c|c|c|}
\hline Valores & Distrito de Saño & Distrito de Ingenio \\
\hline Media aritmética & 14.2 & 13.2 \\
\hline T Student & 2.92 & 2.92 \\
\hline$p$ & 0.004 & 0.004 \\
\hline Nivel de significancia & 0.05 & 0.05 \\
\hline
\end{tabular}

Fuente: elaboración propia

Se comprobó la presencia de diferencias significativas en la comunicación ambiental en individuos de 5 años de los distritos Ingenio y Saño - Huancayo 2017 (tabla 6), entendiéndose como comunicación ambiental el proceso como se transmite desde un punto de vista especializado la información sobre medio ambiente en este caso a individuos de 5 años de edad, reportándose la existencia de diferencias significativas entre las medias aritméticas obtenidas en individuos de 5 años de los dos distritos en estudio.

Muchos estudios sobre percepción ambiental en niños y niñas en edad preescolar han demostrado la baja percepción que estos demuestran ante el ambiente, tal es el caso de un estudio preliminar realizado por Pineda Jiménez et al (2018) en Morelos, México donde comprueban la poca incidencia que la asignatura ambiental en la obtención de una mejor 
formación ambiental en seis escuelas, evidenciándose la necesidad de identificar estrategias educativas más efectivas en esta área.

Tabla 6. Resultados del T student en la comunicación ambiental en niños de 5 años de los Distritos Ingenio y Saño, Huancayo, Perú.

Fuente: elaboración propia

\begin{tabular}{|c|c|c|}
\hline Valores & Distrito de Saño & Distrito de Ingenio \\
\hline Media aritmética & 15.10 & 14.6 \\
\hline T Student & 4.415 & 4.415 \\
\hline$p$ & 0.004 & 0.004 \\
\hline Nivel de significancia & 0.05 & 0.05 \\
\hline
\end{tabular}

Estos resultados coinciden con los de Moreno, Corraliza y Ruiz (2005), los cuales realizaron la investigación: mediación de comportamientos ambientales hacia problemas específicos, los cuales visualizaron que la tarea más relevante para el resguardo de los diferentes ecosistemas y además el mayor desacierto a la hora de solucionar la problemática observada en los diferentes ecosistemas ha sido la aparente imposibilidad para generar un cambio socioambiental.

Moreno, Corraliza y Ruiz (2005), señalan que uno de los inconvenientes para generar esa transformación social es inexcusablemente la percepción de que la sociedad no hace parte de esa discusión, por lo que es común observar que los resultados a través de gestiones individuales no generan resultados efectivos durante la búsqueda de soluciones ambientales. De allí que este estudio confirma que presentar acciones positivas orientadas conscientemente hacia un óptimo mantenimiento de los bienes naturales a través del desarrollo sostenible de los ecosistemas orientados resolver las necesidades sociales e individuales. De manera que, este parece ser un aspecto cuya importancia cobra sentido, además de pertinencia en tanto obedece a la reflexión en el ámbito ambiental.

Cortés (2018), en un estudio realizado en desarrollar una formación ambiental en niños y niñas de 5 a 10 años de edad asociados además con sus padres demostró las inadecuadas prácticas ambientales que se presentaban en una Institución en Colombia, mejorando gracias a la aplicación de programas de formación ambiental, concibiendo una apropiación en la comunidad de estudio con respecto al tema y con además una preocupación en miras de encontrar solución a los problemas ambientales de la zona.

En este orden de ideas se evidencia que para mejorar la percepción ambiental en individuos desde temprana edad es necesaria una reorientación hacia la formación ambiental de estos como lo refiere en su estudio Pérez Payro et al (2017), donde refieren que la creación de una cultura ambiental debe estar encauzada a que todos los funcionarios educativos que forman a los docentes asuman un nivel de conciencia más desarrollado con respecto a la problemática ambiental a nivel mundial, donde no solo se manifieste el querer ser parte activa de su protección sino garantizar de manera responsable la aplicación de nuevas medidas para mitigar ese tipo de problemas.

En resumen, se evidencia que a pesar de reconocer lo complejo del estudio de la cultura ambiental y de percibir el nivel de compromiso moral hacia ellos, las personas son de la 
opinión de que no es sencillo actuar orientados al beneficio del medio ambiente y que en general se actúa menos proambientalmente.

El análisis de esta visión social, los inconvenientes para generar acciones positivas para el beneficio ambiental y la poca consciencia ecológica son claves para garantizar un efectivo trabajo para el desarrollo sostenible de los bienes naturales compartidos. Por su parte Girón y Leyva (2013), llevaron a cabo una investigación donde se analiza la aplicación de la educación ambientalista y su impacto en la percepción, conocimiento y conducta hacia el ambiente basados en los resultados obtenidos en educandos, sus educadores y su familia, que permitieron generar estrategias para la adecuación del proceso educativo por medio del desarrollo de actitudes y comportamientos proambientales.

Estos datos concuerdan con los de la investigación de Álvarez-Garcia et al (2018), en su investigación sobre las competencias ambientales basadas en los planes de estudios en el área educativa, demostrando en los estudiantes la ausencia de competencias ambientales que permitan desarrollar una formación ecológica de altura en el colegio y que permita apoyar al desarrollo de una formación ambiental a sus futuros iniciados. De igual manera determinan, que el obtener este tipo de competencias pareciera estar condicionada por las zonas de estudios, por el sexo, y el hábitat donde se desenvuelva cada persona, además del tipo de actividades en las cuales distribuya su tiempo de ocio relacionado con el medio ambiente.

Según Berenger et al (2002), son varias las investigaciones que han demostrado la existencia de un elevado estado de descontento ambiental con que se muestra hacia los representantes ambientales de casi todos los países y los métodos usados para su protección. Por lo que, el punto de vista del problema debe cambiarse dependiendo de la conciencia ambiental de la sociedad a conocer y así fortalecer los niveles de sensibilidad social.

De la misma manera Rivera-Jacinto y Rodríguez- Ulloa (2009), observaron la percepción, conocimiento y conducta ambiental en estudiantes en este caso universitarios, demostrando que a pesar de poseer actitudes ambientales adecuadas, estos no presentan comportamientos ambientales pertinentes, por lo que es necesario mejorar el enfoque que se le ha dado a la educación ambiental a jóvenes, empezando por los niños desde 5 años, para así fortalecer en los individuos comportamientos ambientales más pertinentes y beneficiosos para el ambiente.

Según Villanueva (2017), es necesario poner en práctica programas de capacitación a docentes para así mejorar su nivel de conocimiento sobre educación ambiental y garantizar la formación de niños con un alto nivel de formación ambiental, que permita por lo tanto mejorar el proceder de los estudiantes desde edades iniciales.

En ese mismo orden de ideas, Otto y Pensini (2017), en su estudio realizado en niños de cuarto a sexto grado de instituciones en Berlin permitió vislumbrar el papel de la formación ambiental en pro de un estilo de vida ecológico por medio de la educación ambiental basada en la naturaleza, ofrecida por instituciones que se centran en la promoción del conocimiento ambiental sobre la flora, la fauna y el ecosistema, a través del contacto real con la naturaleza.

Según lo anteriormente analizado, es procedente decir que la protección de los diferentes ecosistemas a nivel educativo debe ser entendido de una vez por todas, como la salud, la felicidad, la belleza y el dinero entre otros elementos, no son causa si no consecuencias de algo trascendental. Asimismo, el lograr desarrollar una cultura favorable hacia el cuidado 
ambiental es una práctica constante de valores, principios y normas que rigen la vida humana y no como las reglas de conducta moral.

Según el Fondo de las Naciones Unidas para la Infancia UNICEF (2008), los infantes constituyen la principal potencia para generar el cambio a nivel mundial, pues por lo general estos presentan un nivel más elevado de conciencia hacia la defensa de los ecosistemas siendo aún más críticos que los adultos, por lo que la formación ambiental enfocada a estos puede favorecer de forma especial, presentar un punto de vista más crítico y sincero, lo que puede ayudar a generar nuevas soluciones a los problemas ambientales, convirtiéndose en embajadores del cambio que permitan generar cambios en la conducta ambiental de los padres.

Por su parte Pineda Jiménez et al (2018), describen a la escuela y el hogar como entidades donde se pueden formar niños con valores, por lo que, el compromiso para generar niños con una formación ambiental de nivel debe considerarse compartida entre padres e instituciones educativas; por lo que los representantes no deben facultar sólo al maestro para esa labor siendo necesario que estos, se apoyen con actividades que sean practicadas en el hogar para así fortalecer la vida de estos. Por lo que estos investigadores hacen énfasis de que es necesario que las autoridades educativas dirijan más dinero y voluntades para que los docentes en el área ambiental con preparación pedagógica se acerquen más a las comunidades llevando el conocimiento a los lugares más recónditos del planeta.

Es importante generar un cambio en la instrucción ambiental impartida en infantes desde temprana edad que genere cambios positivos en su percepción o actitud hacia la cultura ambiental por medio de la búsqueda de estrategias de enseñanza que así lo permita, en este orden de ideas, Arredondo et al (2017), reconocen que las prácticas fundamentada en textos para la educación ambiental quedan sujetas a malas interpretaciones y a contradicciones entre discursos y prácticas lejanas de las estrategias que fomentan la experiencia directa con la naturaleza las que generan mayor motivación, e interés en los estudiantes.

Otto Siegmar y Pensini (2017), proponen que además de fomentar el conocimiento ambiental, se debe trabajar en la conexión entre la naturaleza y la formación ambiental basados en que la naturaleza proporcione una motivación intrínseca para comportarse de manera ecológica, ofreciendo así un enfoque duradero del comportamiento ecológico, lo que de cierto modo mejoraría las actitudes hacia la cultura ambiental.

El aprendizaje sobre naturaleza en los niños debe iniciarse desde el currículo, la escuela y la vivencia, mediante un conjunto de políticas y estrategias sencillas que desarrolle conciencia ambiental en los futuros ciudadanos.

Para Díaz y Fuentes (2018), el tema ambiental a pesar de ser importante para el desarrollo en la formación ambiental, es un tema que se le ha dedicado poco para su estudio y manejado en el contexto socio-educativo. A pesar de ser un tema elemental para la formación de expertos en ciertas disciplinas de las ciencias naturales y otras afines, desde el ámbito educativo se avizoran escuetas pruebas que demuestren el nivel cultura ambiental y su aplicación en niños de nivel escolar.

Según los resultados obtenidos en los distritos Saño e Ingenio es indispensable generar una serie de cambios en la estrategia educativa instruccional aplicada, para mejorar la actitud ambiental en niñas y niños a través de los maestros, y así generar efectos positivos en su formación ambiental; con respecto a este tema son muchos los investigadores que han generado estudios con ese fin, por ejemplo, Jiménez (2014), en su estudio basado en la 
aplicación de un programa para medir la percepción, comportamiento y manejo sostenible del agua en niños de educación escolar reportó que el programa aplicado generó efectos importantes en la mejora de ese tipo de actitudes hacia el ambiente.

Según Brito y Castillo (2018), las dinámicas educativas en materia ambiental tienen la obligación, además la responsabilidad de educar bajo esta premisa, la escuela juega un papel preponderante en la ejecución, el diseño, la sistematización de temas que tengan que ver con el ambiente. Así mismo, las políticas educativas deben y están en la tarea de coordinar en todos los ámbitos una conciencia ambiental, pensar verde tal y como reza un slogan en una de las organizaciones mundiales que se encarga de proteger el ambiente. En este sentido, pensar ecológicamente es pensar que nuestro planeta es un ser vivo. De allí se desprende que todas nuestras acciones deben estar direccionadas bajo este enfoque. No solo para llevar a cabo actividades intermitentes en torno al ambiente, sino de crear plenamente los esquemas de pensamiento que conlleven a la generación de nuevos y mejores individuos que estén en capacidad de generar en su futuro más remoto una sociedad ambientalmente más armoniosa y saludable.

El objetivo de este artículo fue comprobar si existen diferencias significativas en el comportamiento de los menores provenientes de los Distritos de Ingenio y Saño - Huancayo, Perú, 2017 hacia la cultura ambiental.

\section{Conclusión}

Según el estadístico de t de Student se concluye que existen diferencias significativas en la actitud o disposición hacia la cultura o formación ambiental en los niños y niñas de 5 años del distrito de Ingenio y Saño, - Huancayo, Perú, observándose la disposición hacia una formación ambiental, enmarcada en actitudes más humanas, con valores ambientales, control de ruidos, comunicación ambiental todos con un $p<0,05$ en ambos distritos, por lo que se puede deducir en estos resultados que las actitudes hacia la cultura ambiental en estas dos poblaciones no son uniformes y se necesita seguir trabajando en el cultivo de valores ambientales para una sociedad responsable.

Aun cuando se evidencia resultados disímiles entre las dos instituciones educativas públicas involucradas se demuestra la actitud de los docentes a través de los planes establecidos por el Gobierno Nacional que generar cambios es siempre posible desde la educación; sin embargo, no se puede pensar que esta es la única vía por excelencia para lograr los cambios que se deseen.

Por lo que es necesario destinar todos los medios posibles y voluntades para que los profesionales encargados de formar a los niños en el área ambiental se puedan dirigir a ellos de la manera más efectiva y así generar en ellos un mayor interés en el desarrollo de un ambiente sostenible y en las entidades gubernamentales.

\section{Referencias Bibliográficas}

Ajzen, Icek \& Fishbein, Martin. (1980). Understanding attitudes and predicting social behavior. Englewood Cliffs, Prentice-Hall. Estados Unidos.

Ajzen, Icek \& Madden, Thomas. (1986). Prediction of Goal-Directed Behavior: Attitudes, Intentions, and Perceived Behavioral Control. Journal of Experimental Social 
Psychology. Volumen 22. EEUU. (Pp. 453-474). DOI: https://doi.org/10.1016/00221031(86)90045-4

Álvarez, Pedro y Vega, Pedro. (2009). Actitudes ambientales y conductas sostenibles. Implicaciones para la educación ambiental. Revista de Psicodidáctica, Volumen 14, $N^{\circ}$ 2. España. (Pp. 245-260).

Álvarez-García, Olaya, Sureda-Negre, Jaume y Comas-Forgas, Rubén. (2018). Evaluación de las competencias ambientales del profesorado de primaria en formación inicial: estudio de caso. Enseñanza de las Ciencias. Volumen 36, N 1. España. (Pp. 117-141).

Ángel-Maya, Augusto. (1995). Desarrollo sostenible: aproximaciones conceptuales. Fundación Natura-Unión Internacional para la Conservación de la Naturaleza. Colombia.

Ángel-Maya, Augusto. (2003). La diosa Némesis. Desarrollo sostenible o cambio cultural. (Cuarta Edición) Editorial Corporación Universitaria Autónoma de Occidente. Colombia.

Arredondo, Melina, Saldivar, Antonio, Limon, Fernando. (2017). Estratégias educativas para abordar lo ambiental. Experiencias em escuelas de educación básica em Chiapas. Revista innovación educativa. Volumen 18, № 76. México. (Pp.13-37).

Bayon, Pablo y Morejón, Anisley. (2005). Cultura ambiental y construcción de entornos de producción social en Cuba: Un reto para el siglo 21. Instituto de Filosofía; Ministerio de ciencia Tecnología y medio Ambiente. Extraído de: http://biblioteca.clacso.edu.ar/ar/libros/cuba/if/marx/documentos/22/Cultura\%20ambie ntal\%20y\%20la\%20construcci\%F3n\%20de\%20entornos\%20de....pdf

Berenger, Jaime, Corraliza, José, Moreno, Marta y Rodríguez, Lourdes. (2002). La medida de las actitudes ambientales: propuesta de una escala de conciencia ambiental (Ecobarómetro). Intervención Psicosocial. Volumen 11, № 3. España. (Pp. 349358).

Bleys, Brent, Defloor, Bart, Van Ootegem, Luc \& Verhofstad, Elsy. (2018). The Environmental Impact of Individual Behavior: Self-Assessment Versus the Ecological Footprint. Environment and Behavior. Volumen 50, Nㅜ․ EEUU. (Pp. 187-212).

Brito, Mariana y Castillo, Luis Guillermo. (2018). Gestión ambiental comunitaria para las niñas y niños del consejo popular Carlos Manuel. Avances. Volumen 20, № 3. (Pp. 298-307). Extraído

http://www.ciget.pinar.cu/ojs/index.php/publicaciones/article/view/375/1300

Cortés M., María Fernanda. (2018). Estrategia pedagógica para desarrollar una cultura ambiental con los niños y niñas de 5 a 10 años de edad y padres de familia de la institución educativa departamental nacionalizado de Jerusalén sede Antonio Nariño. Corporación Universitaria uniminuto de Dios. Tesis. Programa Licenciatura en pedagogía infantil Girardot Cundinamarca, Colombia. Extraído de: https://repository.uniminuto.edu/bitstream/handle/10656/7134/T.PED CortesMariaFer nanda_2018.pdf?sequence=18isAllowed=y

De Alba, Alicia, Viesca, Martha, Alcántara, Angélica, Esteban, Norma y Gutiérrez, Margarita. (1993). El libro de texto y la cuestión ambiental. Los contenidos ecológicos en el curriculum de la primaria. Ed. UNAM. México. 
Díaz, Jocelyn. y Fuentes, Fabio. (2018). Desarrollo de la conciencia ambiental en niños de sexto grado de educación primaria. Significados y percepciones. Revista de investigación educativa. Volumen 2. España. (Pp. 136-167).

Fishbein, Martin, \& Aizen, Icek (1975). Belief, attitude, intention, and behavior. Publisher Reading, Mass.: Addison-Wesley. EEUU.

Girón, Mary y Leyva, Juana. (2013), El eje ambiental en la escuela "La Esperanza": un estudio sobre actitudes y comportamientos ambientales. Innovación Educativa. Volumen 13, $\mathrm{N}^{\circ}$ 63. España. (Pp. 117-147).

Hernández-Ayón, Hermilio. \& Hernández-Ayón, Francisco Javier. (2014). Cultura por la vida. En: Cultura Ambiental. Coordinadores: Saldaña-Durán, C. E. y Messina-Fernández, S. R. ECORfan-México. Universidad Autónoma de Nayarit, 2014. Extraído de: https://www.ecorfan.org/manuales/manuales_nayarit/Cultura\%20Ambiental\%20V6.pdf

Holahan, Charles. (2014). Environmental Psychology. New York: Ramdom House. [Traducido al castellano en 1991, Psicología Ambiental: Un enfoque general. México: Limusa]. Human Decision Process. Volumen 50. Estados Unidos. (Pp. 179-211).

Jiménez, Elvira. (2014). Programa Gebisa, Actitudes, Comportamiento y Gestión sostenible del agua en estudiantes del nivel Inicial de Huancayo". Universidad nacional del centro del Perú, Escuela de postgrado unidad de postgrado de la facultad de educación. (Tesis Magister en Educación). Recuperado de: http://repositorio.uncp.edu.pe/bitstream/handle/UNCP/3247/Jimenez\%20Asto.pdf?se quence $=1$ \&isAllowed $=y$

Maldonado, Wilson, Baldiris, Idelfonso y Díaz, Jhon. (2011). Evaluación de la calidad del agua en la Ciénaga de la Virgen (Cartagena, Colombia) durante el período 2006-2010. Revista Científica Guillermo de Ockham. Volumen 9, $N^{\circ} 2$. Colombia. (Pp 79-87).

Moreno, Marta, Corraliza, José Antonio y Ruiz, Juan Pedro. (2005). Escala de actitudes ambientales hacia problemas específicos. Psicothema. Volumen 17. № 3. España. (Pp 502-508).

Otto, Siegmar., \& Pensini, Pamela. (2017). Nature-based environmental education of children: Environmental knowledge and connectedness to nature, together, are related to ecological behaviour. Global Environmental Change. Volumen 47. Países bajos. (Pp 88-94). DOI: https://doi.org/10.1016/j.gloenvcha.2017.09.009

Pérez Payrol, Virginia Bárbara, Baute Rosales, Mireya \& Luque Espinoza de los Montero, Margarita. (2017). La formación de la cultura ambiental en la carrera Ciencias de la Educación. Revista Conrado, Cuba. Volumen 13 (1-Ext), 92-100. Extraído de: https://conrado.ucf.edu.cu/index.php/conrado/article/view/653

Pineda, Citladi., López, Xavier, Wehncke, Elisabet y Maldonado, Belinda. (2018). Construir sociedades comprometidas con el entorno natural: educación ambiental en niños del sur de Morelos, México. Región y sociedad. Volumen 72. México. (Pp. 1-25). DOI: https://doi.org/10.22198/rys.2018.72.a896

Rivera-Jacinto, Marco. y Rodríguez-Ulloa, Carla, (2009). Actitudes y comportamientos ambientales en estudiantes de enfermería de una Universidad Pública del Norte del Perú. Revista Peruana de Medicina Experimental y Salud Pública. Volumen 26, N³. Perú. (Pp. 338-42). 
Roque, Martha. (2003). Una concepción educativa para el desarrollo de una cultura ambiental desde una perspectiva cubana. IV congreso de Iberoamericano de Educación ambiental (Pp. I 29) La Habana. Extraído de: http://www.anea.org.mx/docs/IVCongresolbero-Memorias.pdf

Sánchez, Hugo y Reyes, Carlos. (2015). Metodología y Diseños en la Investigación Científica. Editorial Bussines Suport. Lima.

Severiche-Sierra, Carlos y Acevedo, Rosa. (2013). Las prácticas de laboratorio en las ciencias ambientales. Revista Virtual Universidad Católica del Norte. Volumen 3, $\mathrm{N}^{\circ} 40$. Colombia. (Pp. 191-203).

Severiche-Sierra, Carlos; Gómez-Bustamante, Edna y Jaimes-Morales, José. (2016). La educación ambiental como base cultural y estrategia para el desarrollo sostenible. TELOS. Revista de Estudios Interdisciplinarios en Ciencias Sociales. Volumen 18, $\mathrm{N}^{\circ}$ 2. Venezuela. (Pp. 266-281).

UNICEF (2008). El niño y el ambiente. Documentos de Trabajo del Personal de UNICEF División de Programas Sección de Agua, Medio Ambiente y Saneamiento Número de Serie Temática WES PD-98-001. Editorial UNICEF, Estados Unidos.

Villanueva, Jenny. (2017). Actitudes pro ambientales y conductas pedagógicas sostenibles en profesores del nivel primario. Educare et Comunicare, Volumen 5, $N^{\circ}$ 2. Perú. (Pp. 25-38). 